CERN-TH/97-72

\title{
Anisotropic Correlated Electron Model Associated With the Temperley-Lieb Algebra
}

\author{
Angela Foerster ${ }^{(1, \dagger, a)}$, Jon Links ${ }^{(2, b)}$ and Itzhak Roditi ${ }^{(3, \ddagger, c)}$ \\ (1) Institut für Theoretische Physik, Freie Universität Berlin, \\ WE2, Arnimallee 14, D 14195 Berlin, Germany \\ ${ }^{(2)}$ Department of Mathematics, University of Queensland, \\ Queensland, 4072, Australia \\ ${ }^{(3)}$ Theory Division, CERN, CH-1211 Geneva 23, Switzerland
}

\begin{abstract}
We present an anisotropic correlated electron model on a periodic lattice, constructed from an R-matrix associated with the Temperley-Lieb algebra. By modification of the coupling of the first and last sites we obtain a model with quantum algebra invariance.
\end{abstract}

\footnotetext{
${ }^{\dagger}$ Permanent address: Instituto de Física da UFRGS, Av. Bento Gonçalves, 9500,

Porto Alegre, 91501-970, Brazil

${ }^{\ddagger}$ Permanent address: Centro Brasileiro de Pesquisas Físicas - CBPF, Rua Dr. Xavier Sigaud, 150, 22290-180 - Rio de Janeiro, RJ - Brazil

e-mail address:

${ }^{(a)}$ angela@if.ufrgs.br,${ }^{(b)}$ jrl@maths.uq.oz.au, ${ }^{(c)}$ roditi@cat.cbpf.br.

CERN-TH/97-72
}

April 1997 
Since the discovery of high $T_{c}$ superconductivity there has been a great interest in the area of integrable highly correlated electron systems. Notably for many years the Hubbard and the supersymmetric $t-J$ models, which are both exactly solvable in one dimension, stood as the prototypes for such types of models. Subsequently other correlated electron models have been formulated [1-3].

In this letter we present a new q-deformed integrable electronic model following the approach of [4], which consists of using the Temperley-Lieb (TL) algebra to obtain solutions of the Yang-Baxter equation. In [3] this was achieved utilizing a 4-dimensional module of Lie superalgebra $g \ell(2 / 1)$, here we proceed along the same lines by investigating the corresponding module of $U_{q}(g \ell(2 / 1))$ in order to obtain a representation of the TL algebra.

The procedure adopted in [3] involved a symmetry breaking transformation so that the resulting Hamiltonian, defined on a one-dimensional periodic lattice, was not invariant with respect to $g \ell(2 / 1)$ but rather its even subalgebra $g \ell(2) \otimes u(1)$. For the present situation, the usual impositon of periodic boundary conditions has the effect of also breaking the $U_{q}(g \ell(2)) \otimes u(1)$ symmetry due to the non-cocommutativity of the quantum algebra generators. However we will show, following the methodology of [5-8] that a quantum algebra invariant closed system can be defined by the introduction of an operator coupling the first and last sites into the expression for the Hamiltonian.

Let $\{|x\rangle\}_{x=1}^{4}$ be an orthonormal basis for a four-dimensional $U_{q}(g \ell(2 / 1))$ module $V$. The quantum superalgebra $U_{q}(g \ell(2 / 1))$ obtained by deforming $g \ell(2 / 1)$ has simple generators $\left\{E_{i}^{i}\right\}_{i=1}^{3} U\left\{E_{i+1}^{i}, E_{i}^{i+1}\right\}_{i=1}^{2}$ which act on this module according to

$$
\begin{aligned}
& E_{i}^{i}|j\rangle=-\left(\delta_{j}^{i}+\delta_{4}^{j}\right)|j\rangle ; i=1,2, j=1,2,3,4 \\
& E_{3}^{3}|j\rangle=\left(\frac{1}{2}+\delta_{4}^{j}-\delta_{1}^{j}\right)|j\rangle ; j=1,2,3,4 \\
& E_{2}^{1}|j\rangle=\delta_{3}^{j}|2\rangle ; j=1,2,3,4 \\
& E_{1}^{2}|j\rangle=\delta_{2}^{j}|3\rangle ; j=1,2,3,4 \\
& E_{3}^{2}|1\rangle=E_{3}^{2}|3\rangle=E_{2}^{3}|2\rangle=E_{2}^{3}|4\rangle=0 \\
& E_{3}^{2}|2\rangle=\left[\frac{1}{2}\right]_{q}^{1 / 2}|1\rangle, \quad E_{3}^{2}|4\rangle=\left[\frac{1}{2}\right]_{q}^{1 / 2}|3\rangle \\
& E_{2}^{3}|1\rangle=-\left[\frac{1}{2}\right]_{q}^{1 / 2}|2\rangle, \quad E_{2}^{3}|3\rangle=\left[\frac{1}{2}\right]_{q}^{1 / 2}|4\rangle
\end{aligned}
$$


where

$$
[x]_{q}=\frac{q^{x}-q^{-x}}{q-q^{-1}}, x \in C .
$$

The quantum superalgebra $U_{q}(g \ell(2 / 1))$ carries the following parity

$$
\left[E_{j}^{i}\right]=([i]+[j])(\bmod .2)
$$

where $[1]=[2]=0,[3]=1$, and by consistency the parity of the basis vectors is chosen to be

$$
[|1\rangle]=[|4\rangle]=0 \quad[|2\rangle]=[|3\rangle]=1
$$

Associated to $U_{q}(g \ell(2 / 1))$ there is also a co-product structure

$$
\Delta: U_{q}(g \ell(2 / 1)) \rightarrow U_{q}(g \ell(2 / 1)) \otimes U_{q}(g \ell(2 / 1))
$$

given by

$$
\begin{aligned}
\Delta\left(E_{i}^{i}\right) & =I \otimes E_{i}^{i}+E_{i}^{i} \otimes I \quad i=1,2,3 \\
\Delta\left(E_{j}^{i}\right) & =E_{j}^{i} \otimes q^{\left(E_{1}^{1}-E_{2}^{2}\right) / 2}+q^{-\left(E_{1}^{1}-E_{2}^{2}\right) / 2} \otimes E_{j}^{i} \quad i, j=1,2 \\
\Delta\left(E_{\ell}^{k}\right) & =E_{\ell}^{k} \otimes q^{\left(E_{2}^{2}+E_{3}^{3}\right) / 2}+q^{-\left(E_{2}^{2}+E_{3}^{3}\right) / 2} \otimes E_{\ell}^{k} \quad k, \ell=2,3
\end{aligned}
$$

Everywhere we shall use the graded-tensor product law, defined by.

$$
(a \otimes b)(c \otimes d)=(-1)^{[b][c]}(a c \otimes b d)
$$

Following a strategy analogous to the one employed in ref. [3] to contruct an hermitian Hamiltonian, we now consider the operator

$$
T=|\psi\rangle\langle\psi|
$$

where $|\psi\rangle$ in an unnormalized vector of $V \otimes V$ defined by

$$
\begin{aligned}
|\psi\rangle & =\left(q^{-1 / 2}|4\rangle \otimes|1\rangle+q^{+1 / 2}|1\rangle \otimes|4\rangle\right) \\
& +\left(q^{-1 / 2}|3\rangle \otimes|2\rangle-q^{1 / 2}|2\rangle \otimes|3\rangle\right)
\end{aligned}
$$

and

$$
\begin{aligned}
\langle\psi| & =\left(q^{-1 / 2}\langle 4| \otimes\langle 1|+q^{+1 / 2}\langle 1| \otimes\langle 4|\right) \\
& +\left(-q^{-1 / 2}\langle 3| \otimes\langle 2|+q^{+1 / 2}\langle 2| \otimes\langle 3|\right)
\end{aligned}
$$


A straightforward calculation shows that

$$
T^{2}=\left[2\left(q+q^{-1}\right)\right] T
$$

and, using the fact that $|\psi\rangle$ spans a 1-dimensional submodule of $U_{q}(g \ell(2 / 1))($ see $[3,4])$

$$
\begin{aligned}
& (T \otimes I)(I \otimes T)(T \otimes I)=T \otimes I \\
& (I \otimes T)(T \otimes I)(I \otimes T)=I \otimes T
\end{aligned}
$$

such that $T$ provides a representation of the $T L$ algebra. This can be used to obtain an R-matrix by the transformation [4]

$$
\check{R}(u)=P R(u)=I+\frac{\sinh (u)}{\sinh (\eta-u)} T,
$$

where $\cosh (\eta)=\left(q+q^{-1}\right)$ and $P$ is the $Z_{2}$-graded permutation operator defined by $P(|x\rangle \otimes|y\rangle)=(-1)^{[|x\rangle][|y\rangle]}|y\rangle \otimes|x\rangle, \forall 1 \leq x, y \leq 4$.

It is easy to check that it satisfies the Yang-Baxter equation

$$
(I \otimes \check{R}(u)(\check{R}(u+v) \otimes I)(I \otimes \check{R}(v))=(\check{R}(v) \otimes I)(I \otimes \check{R}(u+v))(\check{R}(u) \otimes I)
$$

A local Hamiltonian can be defined by [9]

$$
H_{i, i+1}=\left.\sinh (\eta) \frac{d}{d u} \check{R}(u)_{i, i+1}\right|_{u=1}=T_{i, i+1},
$$

where on the N-fold tensor product space we denoted

$$
\check{R}(u)_{i, i+1}=I^{\otimes(i-1)} \otimes \check{R}(u) \otimes I^{\otimes(N-i-1)} .
$$

Finally in view of the grading the basis vectors of the module $V$ can be identified with the eletronic states as follows

$$
|1\rangle \equiv|+-\rangle=c_{+}^{+} c_{-}^{+}|0\rangle,|2\rangle \equiv|-\rangle=c_{-}^{+}|0\rangle,|3\rangle \equiv|+\rangle=c_{+}^{+}|0\rangle,|4\rangle \equiv|0\rangle
$$

allowing $H_{i, i+1}$ to be expressed in terms of the canonical fermion operators as

$$
\begin{aligned}
H_{i, i+1} & =q n_{i,+} n_{i,-}\left(1-n_{i+1,+}\right)\left(1-n_{i+1,-}\right)+q^{-1}\left(1-n_{i,+}\right)\left(1-n_{i,-}\right) n_{i+1,+} n_{i+1,-} \\
& +q^{-1} n_{i,+}\left(1-n_{i,-}\right) n_{i+1,-}\left(1-n_{i+1,+}\right)+q n_{i,-}\left(1-n_{i,+}\right) n_{i+1,+}\left(1-n_{i+1,-}\right) \\
& -S_{i}^{+} S_{i+1}^{-}-S_{i}^{-} S_{i+1}^{+}+c_{i,+}^{+} c_{i,-}^{+} c_{i+1,-} c_{i+1,+}+c_{i+1,+}^{+} c_{i+1,-}^{+} c_{i-} c_{i+} \\
& +q c_{i,+}^{+} c_{i+1,+} n_{i,-}\left(1-n_{i+1,-}\right)+\text { h.c. }-c_{i,-}^{+} c_{i+1,-} n_{i,+}\left(1-n_{i+1,+}\right)+\text { h.c. } \\
& +c_{i,-} c_{i+1,-}^{+} n_{i+1,+}\left(1-n_{i,+}\right)+\text { h.c. }-q^{-1} c_{i,+} c_{i+1,+}^{+} n_{i+1,-}\left(1-n_{i,-}\right)+\text { h.c. }
\end{aligned}
$$


where the $c_{i \pm}^{(+)}$are spin up or down annihilation (creation) operators, the $S_{i}^{\prime} s$ spin matrices and the $n_{i}^{\prime} s$ occupation numbers of electrons at lattice site $i$.

Thus we obtain a Hamiltonian describing electron pair hopping, correlated hopping and generalized spin interactions. We notice that in the limit $q \rightarrow 1$ we recover the isotropic Hamiltonian discussed in [3].

By means of the quantum inverse scattering method $[9,10]$ it is possible to show that the model is integrable. Basically, by this procedure, the Hamiltonian (12) is related to the transfer matrix of a graded vertex model [11] (see also [12]), constructed from the spectral parameter dependent R-matrix. The associated Yang-Baxter algebra implies the commutativity of the transfer matrix for different spectral parameters, which reflects the integrability of the model.

The global Hamiltonian takes the form

$$
H=\sum_{i=1}^{N-1} H_{i, i+1}+H_{N 1} .
$$

It is not invariant with respect to $U_{q}(g \ell(2)) \otimes u(1)$ since $H_{N 1} \neq H_{1 N}$ reflecting the noncocommutativity of the co-product. However, by modifying the above Hamiltonian we can obtain a quantum algebra invariant model as follows [c.f. 5- 8].

Let $\sigma$ denote the braid generator defined by

$$
\begin{aligned}
\sigma & =\lim _{u \rightarrow \infty} \check{\mathrm{R}}(u) \\
& =I-e^{-\eta} T
\end{aligned}
$$

which satisfies the braid relations

$$
(\sigma \otimes I)(I \otimes \sigma)(\sigma \otimes I)=(I \otimes \sigma)(\sigma \otimes I)(I \otimes \sigma)
$$

Setting $G=\sigma_{12} \sigma_{23} \cdots \sigma_{N-1 N}$, then it follows from the TL relations that

$$
G H_{i, i+1} G^{-1}=H_{i+1, i+2} \quad, \quad i=1, \cdots N-2
$$

We now define

$$
H_{o}=G H_{N-1, N} G^{-1}
$$

which can be shown to satisfy

$$
G H_{o} G^{-1}=H_{12}
$$


and set our new Hamiltonian to be

$$
H=\sum_{i=1}^{N-1} H_{i, i+1}+H_{o}
$$

satisfying $[H, G]=0$ and additionally invariance with respect to the quantum algebra $U_{q}(g \ell(2)) \otimes u(1)$.

The details about the Bethe ansatz solution of the present model will be developed elsewhere.

\section{ACKNOWLEDGEMENTS}

A.F. would like to thank the Institute für Theoretische Physik - FUB for its kind hospitality, particularly M. Karowski. She also thanks SFB 288 - Differentialgeometrie und Quantenphysik for financial support.

J.L. is supported by an Australian Research Council Postdoctoral Fellowship.

I.R. would like to thank V. Rittenberg for interesting discussions. He also thanks CERN for its warm hospitality and the CNPq for financial support. 


\section{References}

[1] F.H.L. Essler and V.E. Korepin 1992 and K. Schoutens, Phys. Rev. Lett. 68 (1992) 2960; Phys. Rev. Lett. 70 (1993) 73

A. Foerster and M. Karowski, Nucl. Phys. B 408 (1993) 512

R.Z. Bariev, A. Klümper, A. Schadschneider and J. Zittartz, J. Phys. A: Math. Gen. 26 (1993) 1249

I.N. Karnaukhov, Phys. Rev. Lett. 73 (1994) 1130 L. Arrachea and A.A. Aligia, Phys. Rev. Lett. 73 (1994) 2240

J. de Boer, V.E. Korepin and A. Schadschneider, Phys. Rev. Lett. 74 (1995) 789

A.J. Bracken, M.D. Gould, J.R. Links and Y-Z Zhang, Phys. Rev. Lett. 74 (1995) 2768 .

[2] M.D. Gould, K.E. Hibberd, J.R. Links and Y-Z Zhang, Phys. Lett. A 212 (1996) 156.

[3] J. Links, J. Phys. A: Math. Gen. 29 (1996) L69.

[4] M.T. Batchelor and A. Kuniba, J. Phys. A 24 (1991) 2599

R.B. Zhang, J. Math., Phys. 32 (1991) 2605.

[5] P. Martin, Potts Models and Related Problems in Statistical Mechanics, Singapore: World Scientific (1991).

[6] M. Karowski and A. Zapletal, Nucl. Phys. B 419 (1994) 567; J. Phys. A: Math. Gen. 27 (1994) 7419.

[7] H. Grosse, S. Pallua, P. Prester and E. Raschhofer, J. Phys. A: Math. Gen. 27 (1994) 4761.

[8] A. Foerster, J. Phys. A: Math. Gen. 29 (1996) 7625

[9] P. Kulish and E. Sklyanin, Lecture Notes in Physics vol 151, p61 (1982).

[10] L.A. Takhtajan and L.D. Faddeev Russian Math. Surveys 34 (1979) 11

Korepin VE, Bogoliubov NM, Izergin AG Quantum Inverse Scattering Method and Correlation Functions, CUP (1993).

[11] P. Kulish and E.K. Sklyanin, J. Sov. Math. 19 (1982) 1596 
[12] V.V. Bazhanov and A.G. Shadrikov. Theor. Math. Phys. 73 (1987) 1302 